\title{
28 Research Suare \\ Screening of Potential Molecular Targets for Proximal, Mid, and Distal Esophageal Cancers
}

\section{Liping Zhong}

Zhuji People's Hospital of Zhejiang Province

Zeying Guo ( $\nabla$ gzy20181008@163.com )

Zhuji People's Hospital of Zhejiang Province https://orcid.org/0000-0001-7969-0339

Yuefen Pan

Huzhou Central Hospital

\section{Xiaoyong Zhu}

Zhuji People's Hospital of Zhejiang Province

\section{Yurong Chen}

Zhuji People's Hospital of Zhejiang Province

\section{Research article}

Keywords: esophageal cancer, esophageal tumor central location, differentially expressed gene, function analysis

Posted Date: June 16th, 2020

DOl: https://doi.org/10.21203/rs.3.rs-34714/v1

License: (c) (i) This work is licensed under a Creative Commons Attribution 4.0 International License.

Read Full License 


\section{Abstract}

Objective: This study aimed to screen the potential molecular targets for proximal, mid, and distal esophageal cancers.

Methods: The clinical data, RNA-seq data, and survival data for TCGA esophageal cancer were retrieved from UCSC Xena database. The samples with clinical information on the esophageal tumor central location were selected. Differential analyses on different groups (mid vs. proximal, distal vs. proximal, and distal vs. mid) were performed. Following the differentially expressed genes (DEGs) in three groups were analyzed, the common genes were subjected to survival analysis, miRNA prediction, and drug-gene analysis. The specific genes were annotated for functional and module analyses.

Results: Six common genes, namely KCNA1, CCDC196, UNC5CL, CYP3A5, CA10, and REG3A, were analyzed among three comparison groups. The expression of $C A 10$ and $R E G 3 A$ was significantly correlated in the prognosis of patients with esophageal cancer. The distal $v s$. mid groups screened 766 specific DEGs, and the mid $v s$. proximal group screened 99 specific DEGs. Functional analysis showed that the genes of distal vs. mid groups, including $E D N 3, C G A, C C R 9$, and GABRA2, as well as genes in the mid vs. proximal groups, including GCGR, OXTR, and MCHR2, were significantly enriched in neural functions. In the distal vs. proximal groups, there were 314 specific DEGs, including CYP2C8 and OXGR1.

Conclusion: Six common genes may serve as the potential molecular targets for the treatment of all proximal, mid, and distal esophageal cancers.

\section{Highlights}

1. Six common genes might be potential targets for the treatment of esophageal cancers.

2. $C A 10$ and REG3A may be the prognostic factors of esophageal cancer.

3. EDN3, CGA, CCR9, and GABRA2 may serve as targets during the treatment of distal and mid esophageal cancers.

4. GCGR, OXTR, and MCHR2 may be therapeutic targets of mid and proximal esophageal cancers.

5. CYP2C8 and OXGR1 might be used as biomarkers to distinguish the distal and proximal esophageal cancers.

\section{Introduction}

Esophageal cancer is a severe malignancy, which has a fatal outcome in the vast majority of cases due to its extremely aggressive nature [1,2]. Currently, esophageal cancer is the eighth most common incident cancer worldwide, and the sixth leading cause of cancer-related death [3]. Moreover, its incidence is rapidly increasing [4]. Radiotherapy is a commonly used treatment modality in conjunction with chemotherapy as either a definitive or pre-operative treatment for esophageal cancer $[5,6]$. Nevertheless, 
the optimal choice of therapeutic strategy must be individualized, based on the disease progression and comorbidities of patients [7].

The esophagus is a long tubular organ extending from the neck to the upper abdomen, surrounded by various structures $[8,9]$. Esophageal tumors tend to grow and migrate longitudinally along the esophageal wall. Therefore, esophageal cancer is divided into proximal, mid, and distal cancer depending on the tumor position. Generally, surgery plays an important role in achieving locoregional control in patients with mid and distal esophageal cancer [10]. Proximal esophageal cancer is located between the cricopharyngeal muscle and the sternal notch, accounting for less than $5 \%$ of all esophageal cancers [11], the management of which remains controversial. If the tumor invades an adjacent organ or has distant metastases, radiotherapy, chemotherapy, and chemoradiotherapy are the therapeutic options regardless of the tumor positions [10]. Recently, the emerging understanding of the molecular mechanisms underlying carcinogenesis provided screen critical molecular targets, which leaded to the development of drugs that target specific molecular events linked to the progression of many cancers, including esophageal cancer [12]. We speculated that the screening of potential molecular targets in esophageal cancer of different position might guide the treatment of proximal, mid, and distal esophageal cancers.

In this study, we collected the RNA-seq data of esophageal cancer from a public database, and the samples included clinical data of esophageal tumor central location. With analysis of the differentially expressed genes (DEGs) between different tumor positions, we screened several key biomarkers or potential molecular targets for proximal, mid, and distal esophageal cancers.

\section{Materials And Methods}

\section{Data retrieval and preprocessing}

The clinical data, RNA-seq data, mutation data, and survival data from the Cancer Genome Atlas (TCGA) esophageal cancer were retrieved from the University Of California Santa Cruz, UCSC (UCSC) Xena database. The original gene expression value in TCGA was stored in the form of ENSGXXXXXXXXXXXX.X, which was converted into gene symbol by the $R$ biomaRt package [13]. The data not matching the gene symbol were deleted. In the case that the same gene symbol corresponded to multiple ENSG numbers, the average value of multiple ENSGs was taken as the final expression value of this gene symbol. The clinical expression matrix matched to a gene symbol was extracted according to the gene type, and only the mRNA was extracted for subsequent analysis. Additionally, since the mRNA expressed at low levels may interfere with the results of differential analysis, these mRNAs (that were expressed in at least $1 / 4$ th of the samples) were removed.

\section{Clinical information processing}

Normal or healthy samples were removed from the esophageal cancer clinical expression profiles, and only tumor samples were selected. We included these esophageal cancers who had never undergone chemotherapy or radiotherapy. The pathological type of esophageal cancer included in the present study 
was squamous cell carcinoma. Moreover, from the remaining tumor samples, the samples with clinical information of esophageal tumor central location were included, and the samples without clinical information were excluded. The remaining samples were included in the subsequent analysis, and the corresponding expression profile and survival data of these samples were extracted from the total expression profiles and survival data for differential analysis.

\section{Differential analysis}

To investigate the differences in gene expression in esophageal cancer patients with different tumor central locations, edgeR package in R [14] was used to perform differential analyses on different groups (mid vs. proximal, distal vs. proximal and distal vs. mid). Differential analysis was performed based on count value, and the threshold of DEGs for screening was set as $p$-value $<0.05$ and |log fold change (FC)| $>0.585$. The three groups of DEGs obtained were analyzed through Venn diagrams (http://bioinformatics.psb.ugent.be/webtools/Venn/).

\section{Analysis of common DEGs from three groups}

The expression of common genes was validated in three groups using the Logcpm function in edgeR packet, and the results are shown in boxplots. To investigate the prognostic role of common genes in esophageal cancer, survival analysis was performed using these common genes. The survival analysis was based on the standardized data processed by Logcpm function in edgeR and the TCGA clinical survival data. Based on the standardized data, the optimal cutoff was calculated using the R survminer package [15]. The data values higher than the optimal cutoff were considered as high expression and those lower than the optimal cutoff were considered as low expression. Survival package of R [16] was used for survival analysis, and the gene with $p<0.05$ was considered as a significant prognosis-related gene in esophageal cancer.

The R maftools package [17] was used to study the mutations of common genes in esophageal cancer. Statistical analysis was also carried out for common genes. The mutation files were obtained from TCGA Genomic Data Commons (GDC) preprocessed by Mutect.

Additionally, the drug-gene interaction database (DGIdb) [18] was used to analyze the drug-gene interaction pairs of common genes, and the interacting relations were visualized by Cytoscape.

Furthermore, to study the regulatory mechanism of miRNAs in those common genes, miRNA-target interaction was predicted by miRWalk 2.0 [19]. The databases used included miRWalk, Microt4, miRanda, mirbridge, miRDB, miRMap, miRNAMap, Pictar2, PITA, RNA22, RNAhybrid, and Targetscan. The interacting relations, in at least nine databases, were considered as positive relations, and the obtained relations were visualized using Cytoscape.

\section{Analysis of specific DEGs in three groups}

The specific DEGs in three groups were performed for gene ontology biological process (GO-BP) and pathway analyses using Metascape [20]. In addition to functional analysis, Metascape could also 
perform protein-protein interaction (PPI) analysis and MCODE [21] subnetwork mining for the input genes. MCODE can calculate the node information of nodes in the network, and the node with the highest score was a seed.

\section{Results}

\section{Differential analysis}

Principal component analysis (PCA) of the original data in three groups showed that except for one sample, the other samples were relatively clustered; thus, the outlier was deleted for subsequent analysis. Differential analysis between groups analyzed 249 (194 upregulated and 55 downregulated) DEGs in the mid vs. proximal groups; 822 (365 upregulated and 457 downregulated) DEGs in the distal vs. proximal groups; 1174 (353 upregulated and 821 downregulated) DEGs in the distal vs. mid groups. The PCA and volcano plot for three comparison groups are shown in Fig. 1.

\section{Logical analysis of three groups of differential genes}

The six common genes among the three comparison groups were analyzed. These genes are potassium voltage-gated channel subfamily A member 1 (KCNA1), coiled-coil domain containing 196 (CCDC196), unc-5 family C-terminal like (UNC5CL), cytochrome P450 family 3 subfamily A member 5 (CYP3A5), carbonic anhydrase 10 (CA10) and regenerating family member 3 alpha (REG3A) (Fig. 2A). The boxplots for six genes are shown in Fig. 2B. Additionally, there were 99 specific genes in the mid $v s$. proximal groups, 314 specific genes in the distal vs. proximal groups, and 766 specific genes in the distal vs. mid groups.

\section{Mutation analysis of six common genes in esophageal cancer}

In terms of the overall genetic mutations in esophageal cancer, missense mutation was the main variant type in esophageal cancer (Fig. 3A). A missense mutation is a base substitution mutation that results in changes in the amino acid sequence of a polypeptide or the base sequence of functional RNA, most of which have harmful or lethal effects. Analysis of the mutation of six common genes showed that KCNA1, $C A 10$, and REG3A had mutation data in TCGA esophageal cancer, and all the variant types were missense mutations (Fig. 3B).

\section{Survival analysis of six common genes in esophageal cancer}

Survival analysis showed that among the six common genes, CA10 and REG3A had a significant effect on the prognosis of patients with esophageal cancer $(\mathrm{p}<0.05)$. Low expression of $C A 10$ and high expression of REG3A had a good prognosis of esophageal cancer (Fig. 4). 


\section{miRNA and drug prediction for six common genes}

According to the screening conditions described in the method section, three genes, namely UNC5CL, $K C N A 1$, and $C A 10$, had shown a strong miRNA-target regulatory relation (Fig. 5A). Additionally, three genes, namely $C Y P 3 A 5, K C N A 1$, and $C A 10$, were predicted to have drug-gene interactions. Nifedipine had interactions with both $C Y P 3 A 5$ and $K C N A 1$. Guanidine hydrochloride and dalfampridine also had interaction with $K C N A 1$ (Fig. 5B).

\section{Function and pathway analyses of specific genes}

A total of 99 specific genes in the mid $v s$. proximal groups were enriched in GOs associated with axonemal dynein complex assembly, positive regulation of synaptic transmission, central nervous system neuron differentiation, and the pathway of neuroactive ligand-receptor interaction. The 314 specific genes in the distal vs. proximal groups were enriched in GOs associated with digestion, regulation of neuron differentiation, negative regulation of the Wnt signaling pathway, and regulation of cell cycle checkpoint, as well as pathways of protein digestion and absorption, and sphingolipid metabolism. The 766 genes specific to the distal vs. mid groups were enriched in GO related to cornification, digestion, regulation of intestinal cholesterol absorption, neurotransmitter transport, and pathways of neuroactive ligand-receptor interaction, fat digestion and absorption, and SLC-mediated transmembrane transport (Fig. 6).

\section{PPI analysis}

The PPI network was constructed based on the 99 specific genes in the mid $v s$. proximal groups, and a subnetwork module, including four genes (glucagon receptor (GCGR), oxytocin/neurophysin I prepropeptide (OXT), oxytocin receptor (OXTR) and melanin-concentrating hormone receptor $2(M C H R 2)$ ), was extracted. From the PPI network constructed by 314 specific genes in distal vs. proximal groups, five modules were extracted. Cytochrome P450 family 2 subfamily $\mathrm{C}$ member 8 (CYP2C8), oxoglutarate receptor 1 (OXGR1), ZFP28 zinc finger protein (ZFP28), collagen type II alpha 1 chain (COL2A1), and urotensin 2 (UTS2) were the seed genes. In the PPI network constructed by 766 specific genes in distal vs. mid groups, 11 modules were extracted, and 11 seeds such as endothelin 3 (EDN3), glycoprotein hormones, alpha polypeptide (CGA), C-C motif chemokine receptor 9 (CCR9), gamma-aminobutyric acid type a receptor alpha2 subunit (GABRA2), and cholinergic receptor nicotinic alpha 4 subunit (CHRNA4) were screened (Fig. 7).

\section{Discussion}

The proximal, mid, and distal esophageal cancers have different biological characteristics. Therefore, surgical and radiation treatment options are usually different. In this study, we selected the DEGs among the three types of esophageal cancers to screen the targeted molecules for the treatment of esophageal cancers. After analysis, six genes were screened to be differentially expressed in all three groups. 
Additionally, hundreds of DEGs specific to three comparison groups were also analyzed. These genes may serve as potential therapeutic targets for esophageal cancers.

In this study, six genes were differentially expressed among the three groups. KCNA1 and CYP3A5 were regulated by nifedipine, which is the most common kind of calcium channel blocker and is used to treat high blood pressure and angina. Especially, calcium channel blockers were recently reported to be associated with cancer [22]. A previous study has reported that cancer cells that have DNA mismatch repair deficiency are resistant to many cytotoxic drugs. Calcium channel blockers may inhibit the pathways that cause drug-resistance [23]. Thus, we speculated that KCNA1 and CYP3A5 might be therapeutic targets of esophageal cancer.

$C A 10$ and REG3A were significantly correlated in the prognosis of patients with esophageal cancer. The CA10 gene encodes a protein belonging to the carbonic anhydrase family of zinc metalloenzymes. Hypoxia and acidosis are prominent features of many tumors, leading to a different metabolism from normal or healthy cells. Two of the simplest metabolic products, protons, and bicarbonate, are produced by the catalytic activity of the carbonic anhydrase [24]. Carbonic anhydrase IX is considered as a molecular marker that may predict the prognosis of renal cancer [25]. REG3A is a member of the REG protein family, which has been demonstrated to be involved in the development of some digestive tumors, including gastric cancer, hepatocellular carcinoma, and colorectal cancer [26]. However, its role in esophageal cancer has not yet been reported.

For the other two genes UNC5CL and CCDC196, UNC5CL was found to be regulated by miR-337-3p and miR-512-5p. miR-337 has been reported to distinguish esophageal squamous cell carcinoma patients from healthy controls [27]. Interestingly, a recent study reported that rs10484761 loci, located in a region of 200-kb on chromosome 6p21, near UNC5CL gene, was a novel susceptible locus for esophageal squamous cell carcinoma. CCDC196 belongs to coiled-coil domain-containing proteins that are directly associated with migratory, invasive, and metastatic phenotypes of cancer cells [28-30]. The role of CCDC196 in esophageal cancer is unknown. Taken together, given the significant differential expression of the KCNA1, CYP3A5, CA10, REG3A, UNC5CL, and CCDC196 among three tumor locations, we speculate that these genes might serve as the potential molecular targets for the treatment of all proximal, mid, and distal esophageal cancers. Additionally, CA10 and REG3A might also be potential prognostic factors of esophageal cancer.

In addition to common genes found in the three groups, there were also some genes specific to each position of esophageal cancers. The distal $v s$. mid groups had the most specific DEGs (766), which suggested that there may exist large differences between the two positions. Mid vs. proximal groups only screened 99 specific DEGs. Interestingly, functional analysis showed that the seed genes of distal $v s$. mid groups (including EDN3, CGA, CCR9, and GABRA2), as well as genes in mid vs. proximal group (such as GCGR, OXTR, and MCHR2), were significantly enriched in neural functions, including neuroactive ligandreceptor interaction, transmission of nerve impulse, and neuronal system. Emerging evidence has suggested that nervous microenvironment plays a critical role in cancer progression [31-33]. Quail et al. 
[34] have reported that nervous microenvironment is an important factor in the early stage of invasion and metastasis of pancreatic cancers. Therefore, treatment targeted at neuro-microenvironment may provide a novel strategy to prevent the progression of some cancers.

Nevertheless, the role of the neuronal system in esophageal cancer has not been fully studied. We speculate that $E D N 3, C G A, C C R 9$, and GABRA2 might serve as potential targets during the treatment of distal and mid esophageal cancers. Additionally, GCGR, OXTR, and MCHR2 may be therapeutic targets of mid and proximal esophageal cancers.

In the distal vs. proximal groups, there were 314 specific DEGs, including CYP2C8 and OXGR1. CYP2C8 encodes a member of the cytochrome P450 superfamily of enzymes, which play essential roles in the metabolism of endogenous and exogenous molecules [35]. The local expression of cytochrome P450s is essential for cancer management since these functionally associated enzymes might be involved in determining the anticancer drug sensitivity [36]. OXGR1 belongs to the $\mathrm{G}$ protein-coupled receptor superfamily, and it is frequently found to be hypermethylated in hepatocellular carcinoma [37]. A recent study revealed that overexpression of $O X G R 1$ promoted prostate cancer development [38]. Thus, we speculated that CYP2C8 and OXGR1 might be used as biomarkers to distinguish between distal and proximal esophageal cancers.

Although the genes screened from our analyses are promising candidate genes, we have not validated these genes through in vivo or in vitro experiments. Therefore, we will further validate and strengthen our findings by developing animal models and collecting clinical samples.

In conclusion, KCNA1, CYP3A5, CA10, REG3A, UNC5CL, and CCDC196 may serve as the potential molecular targets for the treatment of all proximal, mid, and distal esophageal cancers. CA10 and REG3A may be prognostic factors of esophageal cancer. $E D N 3, C G A, C C R 9$, and GABRA2 may serve as potential targets during the treatment of distal and mid esophageal cancers. GCGR, OXTR, and MCHR2 may be therapeutic targets of mid and proximal esophageal cancers. CYP2C8 and OXGR1 may be used as biomarkers to distinguish between distal and proximal esophageal cancers.

\section{Declarations}

\section{Ethics approval and consent to participate}

Not Applicable.

\section{Consent for publication}

Not applicable.

\section{Competing Interests}

The authors declare that no conflicts of interest exist. 


\section{Funding}

This work was supported by the Science Technology Projects of Zhejiang Province (No. 2017C33207).

\section{Authors' Contributions}

All authors participated in the conception and design of the study.

Conceived and drafted the manuscript: Liping Zhong and Zeying Guo;

Analyzed the data: Yuefen Pan and Yurong Chen;

Drew figures: Xiaoyong Zhu;

Wrote the paper: Liping Zhong and Zeying Guo;

Han shuwen takes full responsibility for the work.

All authors read and approved the paper.

\section{Acknowledgements}

The authors gratefully acknowledge the multiple databases, which made the data available.

\section{Availability of data and materials}

The datasets generated during the current study are not publicly available but obtained from corresponding authors on reasonable request.

\section{References}

1. Li L, et al. The candidate tumor suppressor gene ECRG 4 inhibits cancer cells migration and invasion in esophageal carcinoma. Journal of Experimental Clinical Cancer Research. 2010;29(1):133.

2. Umar SB, Fleischer DE. Esophageal cancer: epidemiology, pathogenesis and prevention. Nature Reviews Gastroenterology Hepatology. 2008;5(9):517.

3. Ferlay J, et al. Cancer incidence and mortality worldwide: sources, methods and major patterns in GLOBOCAN 2012. International journal of cancer. 2015;136(5):E359-86.

4. Pennathur A, et al. Oesophageal carcinoma. The Lancet. 2013;381(9864):400-12.

5. Minsky BD, et al. INT 0123 (Radiation Therapy Oncology Group 94 - 05) phase III trial of combinedmodality therapy for esophageal cancer: high-dose versus standard-dose radiation therapy. Journal of clinical oncology. 2002;20(5):1167-74.

6. van Hagen P, et al. Preoperative chemoradiotherapy for esophageal or junctional cancer. $\mathrm{N}$ Engl $\mathrm{J}$ Med. 2012;366(22):2074-84. 
7. Jang R, Darling G, Wong RK. Multimodality approaches for the curative treatment of esophageal cancer. J Natl Compr Canc Netw. 2015;13(2):229-38.

8. Dieleman EM, et al. Four-dimensional computed tomographic analysis of esophageal mobility during normal respiration. International Journal of Radiation Oncology* Biology* Physics. 2007;67(3):77580.

9. Qiu G, et al. Differences in displacement of the proximal and distal ends of mid-upper thoracic esophageal squamous cell carcinoma. Molecular clinical oncology. 2016;5(1):143-7.

10. Sohda M, Kuwano H. Current Status and Future Prospects for Esophageal Cancer Treatment. Annals of Thoracic \& Cardiovascular Surgery Official Journal of the Association of Thoracic \& Cardiovascular Surgeons of Asia, 2016. 23(1): p. 1.

11. Mendenhall WM, et al. Management of cervical esophageal carcinoma. in Seminars in radiation oncology. 1994: Elsevier.

12. Belkhiri A, El-Rifai W. Advances in targeted therapies and new promising targets in esophageal cancer. Oncotarget. 2015;6(3):1348.

13. Durinck $S$, et al. BioMart and Bioconductor: a powerful link between biological databases and microarray data analysis. Bioinformatics. 2005;21(16):3439-40.

14. Robinson MD, McCarthy DJ, Smyth GK. edgeR: a Bioconductor package for differential expression analysis of digital gene expression data. Bioinformatics. 2010;26(1):139-40.

15. Kassambara A, Kosinski M. Survminer: drawing survival curves using 'ggplot2'R package version 0.4. 0. 2017. 2017.

16. Therneau TM, Grambsch PM, Modeling survival data: extending the Cox model. 2013: Springer Science \& Business Media.

17. Mayakonda A, et al. Maftools: efficient and comprehensive analysis of somatic variants in cancer. Genome research. 2018;28(11):1747-56.

18. Griffith M, et al. DGIdb: mining the druggable genome. Nature methods. 2013;10(12):1209.

19. Dweep H, Gretz N. miRWalk2. 0: a comprehensive atlas of microRNA-target interactions. Nature methods. 2015;12(8):697.

20. Zhou Y, et al. Metascape provides a biologist-oriented resource for the analysis of systems-level datasets. Nature communications. 2019;10(1):1523.

21. Bandettini WP, et al. MultiContrast Delayed Enhancement (MCODE) improves detection of subendocardial myocardial infarction by late gadolinium enhancement cardiovascular magnetic resonance: a clinical validation study. J Cardiovasc Magn Reson. 2012;14(1):83.

22. Li W, et al. Calcium channel blockers and risk of breast cancer: a meta-analysis of 17 observational studies. PloS one. 2014;9(9):e105801.

23. Li VA, et al. Near-infrared light responsive folate targeted gold nanorods for combined PhotothermalChemotherapy of Osteosarcoma. ACS Appl Mater Interfaces. 2017;9(16):14453-69. 
24. Cobley CM, et al. Gold nanostructures: a class of multifunctional materials for biomedical applications. Chem Soc Rev. 2011;40(1):44-56.

25. Wang $S$, et al. Highly efficient capture of circulating tumor cells by using nanostructured silicon substrates with integrated chaotic micromixers. Angew Chem Int Ed. 2011;50(13):3084-8.

26. Li J, et al. Comparison of bare and amino modified mesoporous silica@ poly (ethyleneimine) s xerogel as indomethacin carrier: Superiority of amino modification. Materials Science Engineering: C. 2016;59:710-6.

27. Cao M, et al. Gadolinium (III)-chelated silica nanospheres integrating chemotherapy and photothermal therapy for cancer treatment and magnetic resonance imaging. ACS Appl Mater Interfaces. 2015;7(45):25014-23.

28. Pannerec-Varna $M$, et al. In vivo uptake and cellular distribution of gold nanoshells in a preclinical model of xenografted human renal cancer. Gold Bulletin. 2013;46(4):257-65.

29. Day ES, et al. Nanoshell-mediated photothermal therapy improves survival in a murine glioma model. Journal of neuro-oncology. 2011;104(1):55-63.

30. Hu X, et al. A microRNA expression signature for cervical cancer prognosis. Cancer research. 2010;70(4):1441-8.

31. Zhao C-M, et al., Denervation suppresses gastric tumorigenesis. Science translational medicine, 2014. 6(250): p. 250ra115-250ra115.

32. Scanlon CS, et al. Galanin modulates the neural niche to favour perineural invasion in head and neck cancer. Nature communications. 2015;6:6885.

33. Venkatesh HS, et al. Neuronal activity promotes glioma growth through neuroligin-3 secretion. Cell. 2015;161(4):803-16.

34. Quail DF, Joyce JA. Microenvironmental regulation of tumor progression and metastasis. Nature medicine. 2013;19(11):1423.

35. Sheweita SA. Drug-metabolizing enzymes mechanisms and functions. Curr Drug Metab. 2000;1(2):107-32.

36. Michael M, Doherty MM. Drug metabolism by tumours: its nature, relevance and therapeutic implications. Expert Opin Drug Metab Toxicol. 2007;3(6):783-803.

37. Lu CY, et al. Aberrant DNA methylation profile and frequent methylation of KLK10 and OXGR1 genes in hepatocellular carcinoma. Genes Chromosom Cancer. 2009;48(12):1057-68.

38. Järvinen S. OXGR1 overexpression as putative prostate cancer promoting factor. 2017.

\section{Figures}



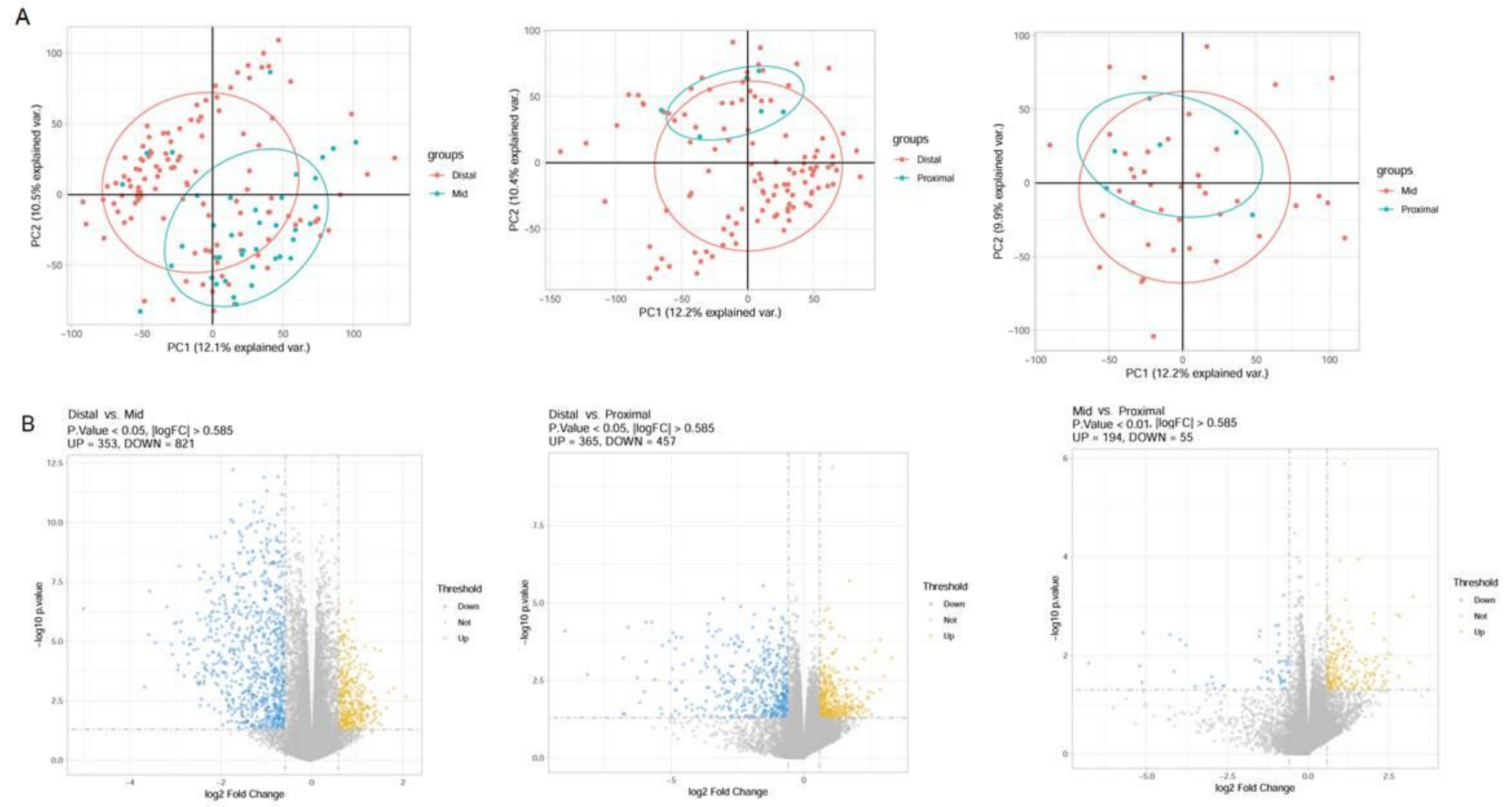

\section{Figure 1}

The principal component analysis (PCA) (A) and a volcano plot (B) for three comparison groups. In volcano plots, yellow represents upregulated genes, and blue represents downregulated genes.

A

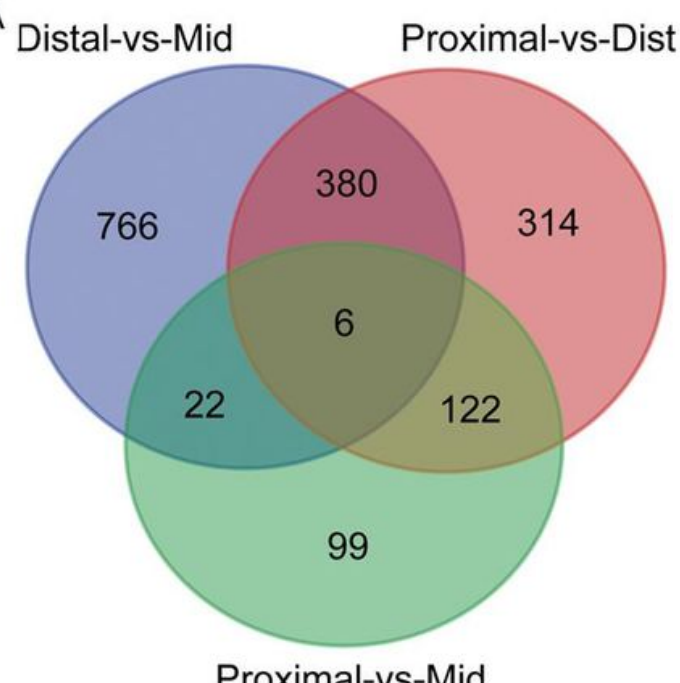

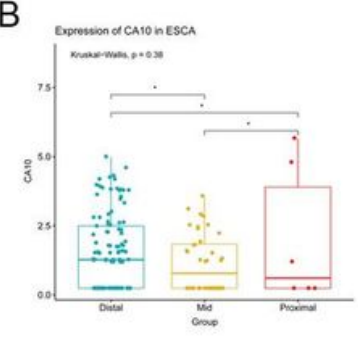

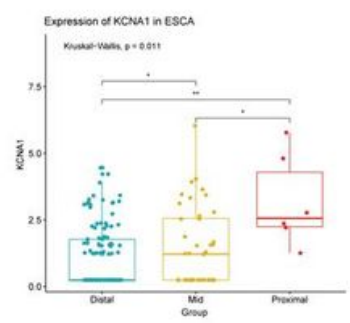

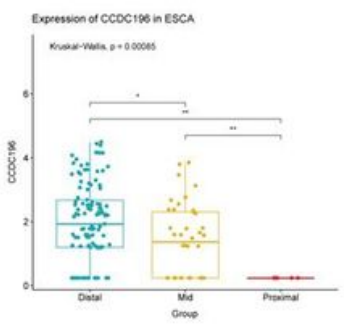
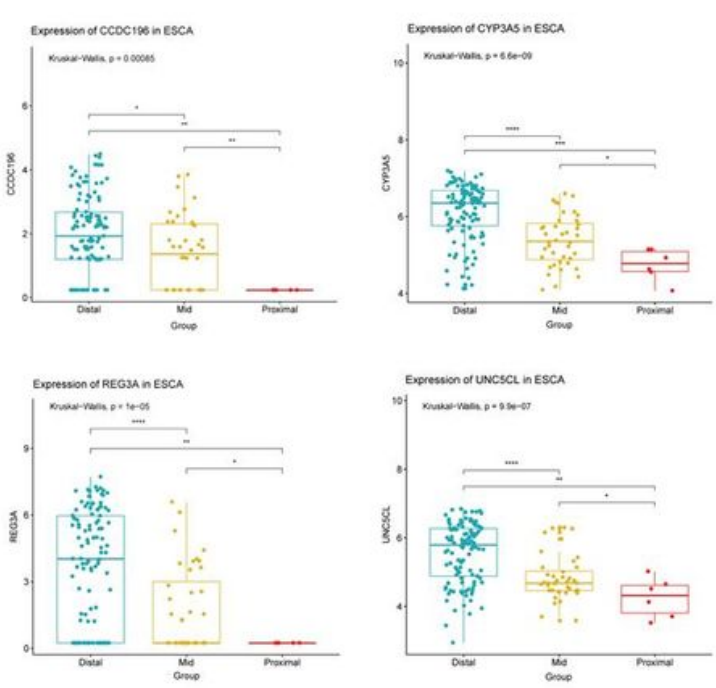

\section{Figure 2}

A: Logical analysis results of three groups of differentially expressed genes. B: The boxplots of six differentially expressed genes in three different positions of esophageal cancers. 
A
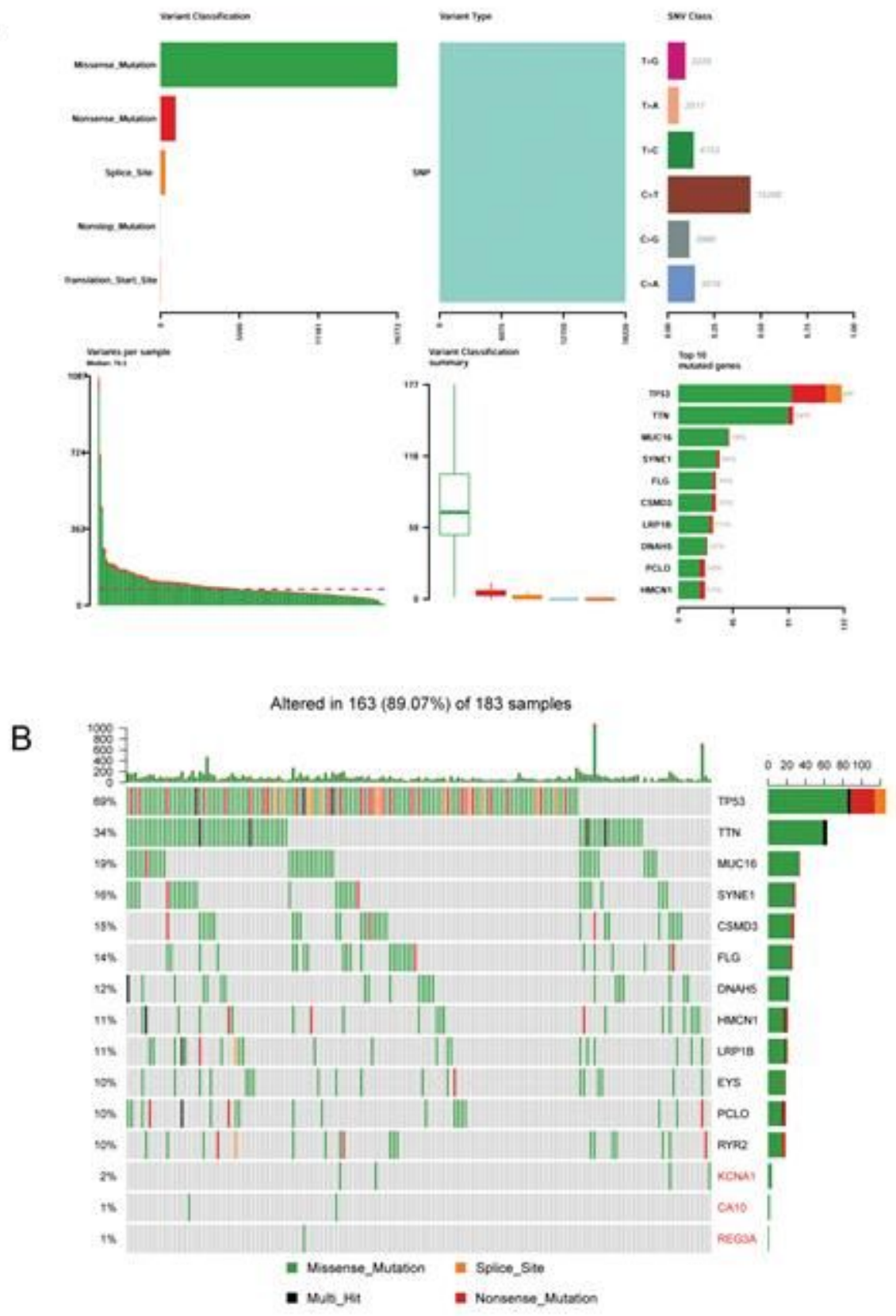

\section{Figure 3}

A: Overall genetic mutations in esophageal cancer. B: Analysis of mutations of six genes in esophageal cancer. Red represents the significantly differentially expressed genes, and black represents the top 10 mutations in esophageal cancers. 

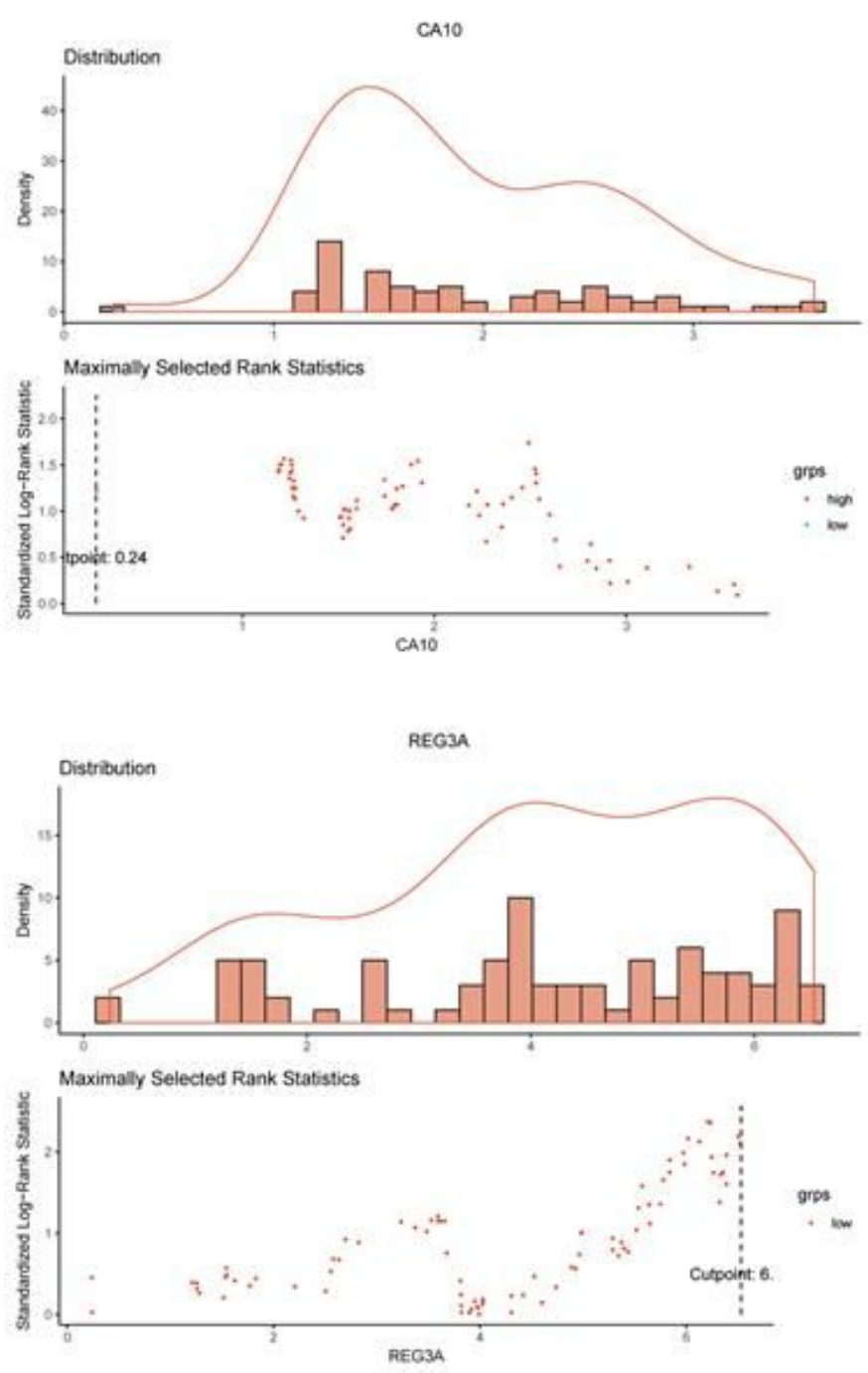
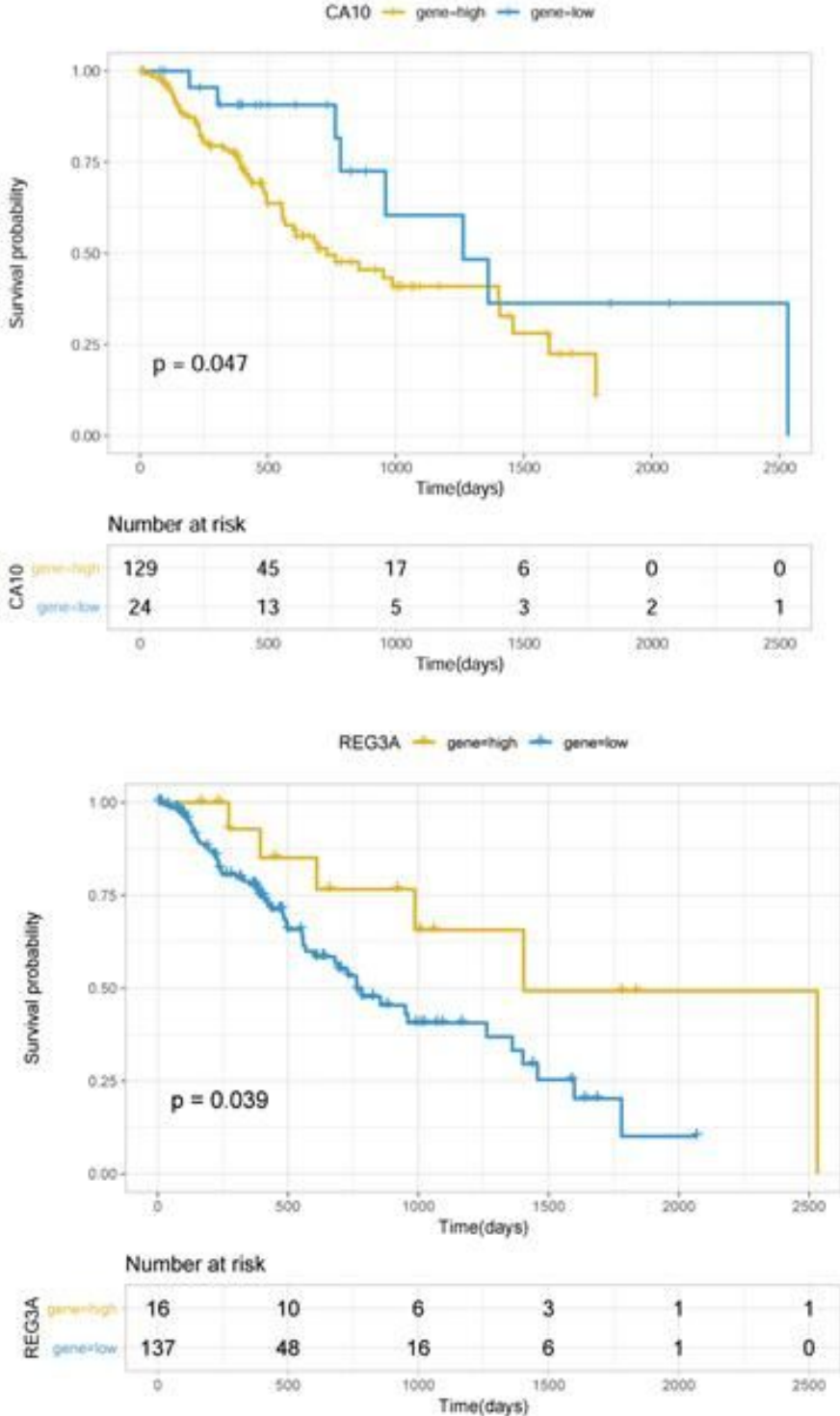

Figure 4

Survival analysis results of CA10 and REG3A in esophageal cancer. The panel on the left is the optimal cutoff value calculated by the survminer, and the panel on the right is the result of survival analysis based on the optimal cutoff. 


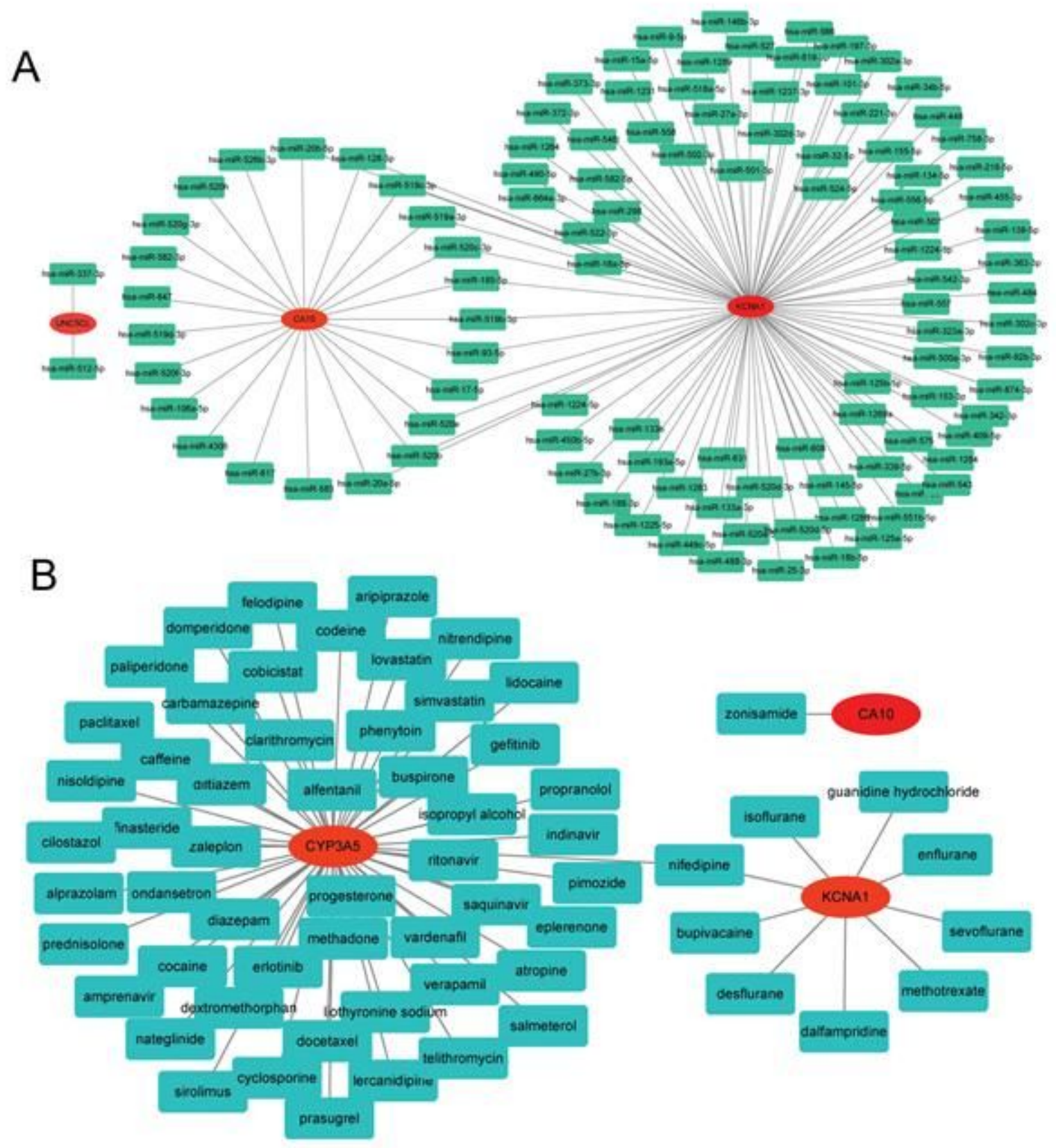

\section{Figure 5}

A: miRNA-target prediction results from miRWalk 2.0. Red represents mRNA, and the green represents miRNA. B: Drug-gene prediction results of six common genes. Red and blue represent genes and drugs, respectively. 
Distal vs. Mid
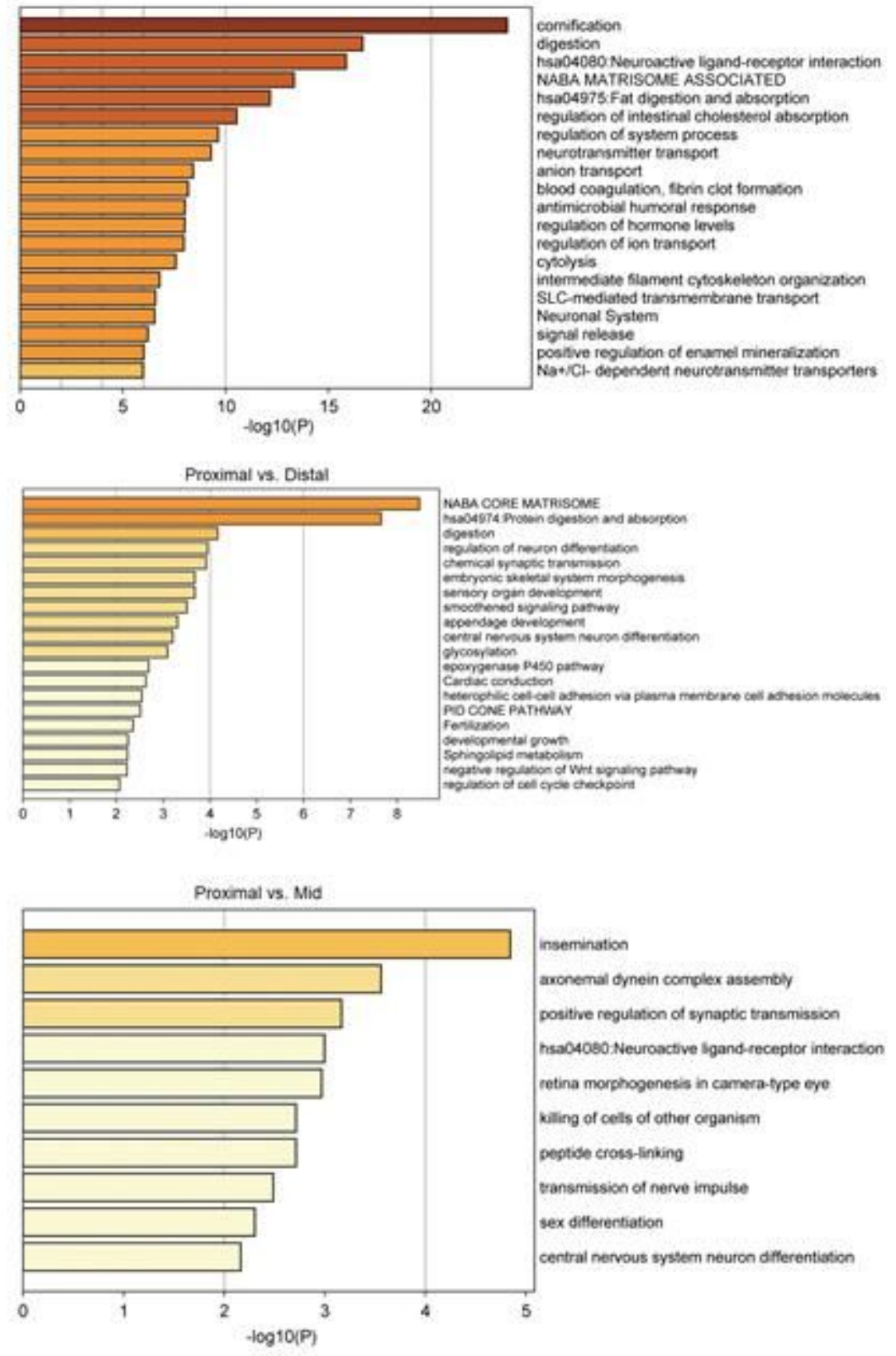

\section{Figure 6}

The biological processes and pathways enriched by differentially expressed genes in three comparison groups. 

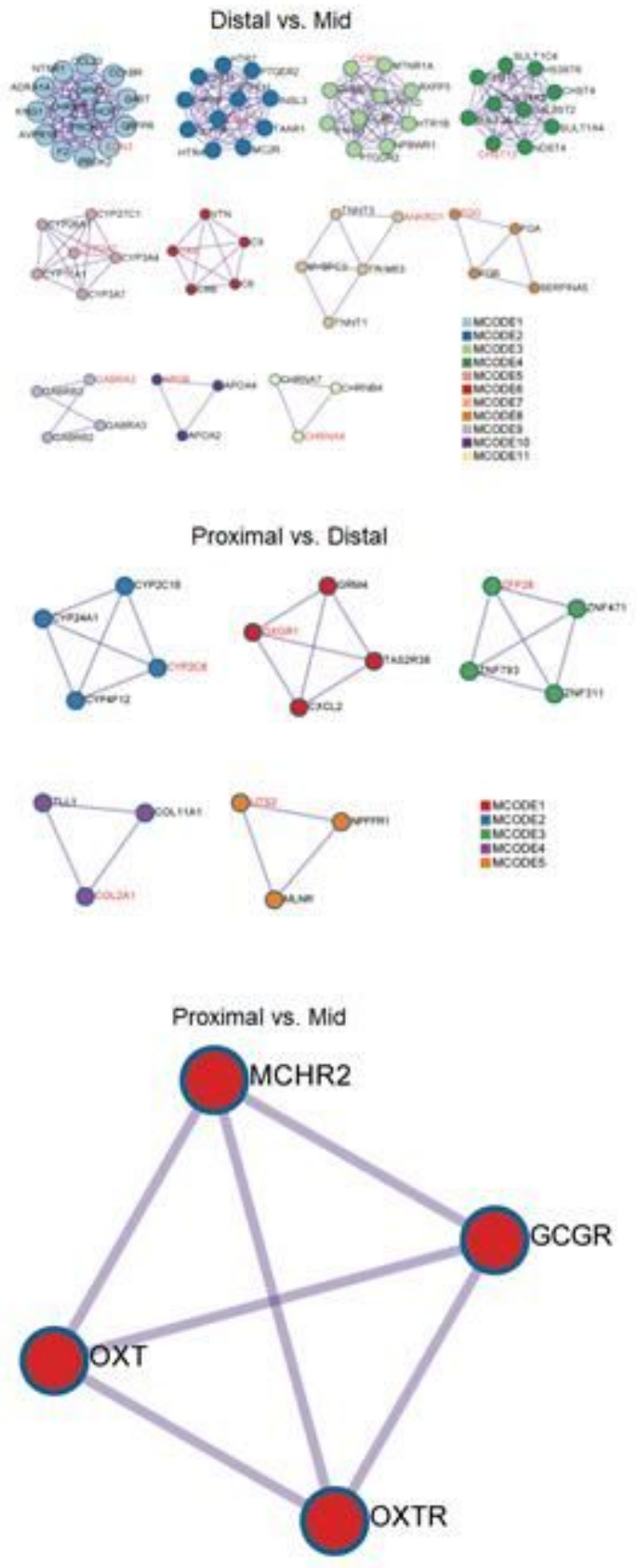

\section{Figure 7}

The sub-network modules were extracted from protein-protein interaction networks. Gene in red represents the seed gene. 